\title{
Éditorial La recherche à Sainte-Justine : pour grandir en santé

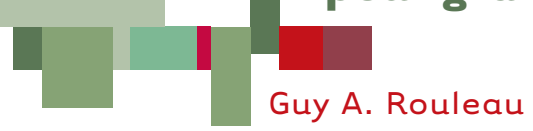



«Un homme n'est jamais si grand que lorsqu'il est à genoux pour aider un enfant»

Pythagore

> 2007 est une année phare et charnière pour notre établissement. Phare car Sainte-Justine fête cette année cent ans au service de la mère et de l'enfant: la modeste clinique fondée par deux femmes de cœur, Justine Lacoste-Beaubien et Irma Levasseur, est aujourd'hui devenue le seul centre hospitalier universitaire mère-enfant québécois, le plus grand au Canada et le deuxième en importance en Amérique du Nord. Grâce au soutien de la direction de l'Hôpital, de l'Université de Montréal et de la Fondation de l'Hôpital Sainte-Justine, le Centre de recherche poursuit une unique mission: acquérir des connaissances qui visent à améliorer la santé et la vie de la mère et de l'enfant.

Année charnière pour le Centre de recherche qui doit se redéfinir à l'aube du XXI siècle. Les progrès spectaculaires de la recherche en protéomique et en génomique ouvrent la voie à une médecine adaptée au profil génétique du patient afin d'apporter une réponse thérapeutique ciblée à ses problèmes de santé. Afin de mieux exploiter les retombées de cette nouvelle approche, six thématiques prioritaires de recherche ont été définies autour des axes d'excellence sur lesquels s'articulera le développement futur du Centre de recherche et de l'Hôpital. Ces axes de force concernent les maladies musculosquelettiques et les sciences du mouvement, les maladies du cerveau, les pathologies fœto-maternelles et néonatales, les maladies virales, l'immunologie et les cancers, les désordres métaboliques et le devenir en santé. L'adéquation entre ces thématiques de recherche prioritaires et les axes d'excellence sur le plan des activités cliniques, constitue une des spécificités de notre institution et assure un transfert efficace des connaissances du laboratoire jusqu'au lit des patients.

L'expertise des chercheurs en maladies musculo-squelettiques et en sciences du mouvement est reconnue mondialement. Ces thématiques favorisent une approche multidisciplinaire, asso- ciant la recherche fondamentale, la recherche clinique et la réadaptation au transfert des connaissances et à l'introduction d'innovations cliniques pour le mieux-être des enfants. L'exemple de la scoliose idiopathique (voir les articles de CarlÉric Aubin et al. et Kareen Letellier et al.) $(\rightarrow) \quad(\rightarrow)$ p. 904 illustre parfaitement l'approche transversale de et 910 de ce ces chercheurs, particulièrement avec la mise au numéro point d'un test diagnostique. Leur objectif est de développer des traitements innovateurs les moins invasifs possible pour permettre aux enfants et aux adolescents de vivre avec le minimum de limitation physique et de dépister les maladies articulaires dès le jeune âge afin de prévenir les séquelles de ces maladies à l'âge adulte.

Le développement de l'axe des maladies du cerveau, regroupant des chercheurs en neurosciences et en psychopathologies développementales, a permis la mise en place de collaborations bio-psycho-sociales en exploitant les données provenant d'études longitudinales. Les scientifiques de cette spécialité ont acquis une reconnaissance internationale, en particulier dans le domaine du développement des systèmes sensoriels (voir l'article de Madeleine Fortin et al.) $(\rightarrow)$, l'étiologie et $(\rightarrow)$ p. 917 le traitement de l'épilepsie (voir les articles de Maryse Lassonde et al. et Lionel Carmant) $(\rightarrow)$ et $\quad(\rightarrow)$ p. 923 le développement du langage chez l'enfant (voir et 929 l'article d'Ann Sutton et al.) $(\rightarrow)$. En outre, les $(\rightarrow)$ p. 934 recherches dans le domaine des psychopatho-

logies et du comportement de l'enfant ont mené à la création d'une unité internationale Inserm ${ }^{1}$ et du Centre d'excellence pour le développement des jeunes enfants. En neurosciences, les chercheurs ont obtenu de nombreuses subventions d'envergure afin d'accroître les approches à grande échelle pour l'étude des maladies neuro-développementales telles que la schizophrénie, l'autisme ou le retard mental.

L'étude des pathologies fœto-maternelles et néonatales met à profit la structure unique du CHU Sainte-Justine pour

${ }^{1}$ Il s'agit d'une Unité Internationale Inserm sur l'étude et la prévention du trouble des conduites chez l'enfant et l'adolescent. 
l'étude des anomalies du développement embryonnaire, fœtal et néonatal. La périnatalogie est un domaine dans lequel le CHU Sainte-Justine s'illustre parmi les centres hospitaliers universitaires les plus dynamiques au Canada. Nos chercheurs s'intéressent à divers aspects de la santé fœto-maternelle dont certains sont illustrés dans le présent numéro, tels que la rétinopathie du prématuré (voir l'article de Martin Leduc et al.) $(\rightarrow)$, le paradoxe cardiovasculaire de la grossesse (voir l'article de Jean St-Louis et al.) $(\rightarrow)$, le flux dans l'isthme aortique (voir l'article de Jean-Claude Fouron et al.) $(\rightarrow)$ et l'effet des antidépresseurs sur la grossesse (voir l'article d'Anick Bérard et al.) $(\rightarrow)$.

$(\rightarrow)$ p. 939

$(\rightarrow)$ p. 944

$(\rightarrow)$ p. 950

$(\rightarrow)$ p. 957 Le CHU Sainte-Justine est un leader reconnu dans le domaine des maladies virales, immunitaires et du cancer, notamment pour la leucémie lymphoblastique aiguë (voir l'article de Marc Ansari et al.) $(\rightarrow)$, l'oncogénétique (voir l'article de Daniel Sinnett et al.) $(\rightarrow)$, la domiciliation des cellules souches dans la moelle osseuse (voir l'article de Sonia F. Desrosiers et al.) $(\rightarrow)$, le rôle du foie dans le système immunitaire (voir l'article de Pascal Lapierre et al.) $(\rightarrow)$, et pour la transmission du VIH de la mère à l'enfant (voir l'article de Doris G. Ransy et al.) $(\rightarrow)$. Notre centre de greffe de sang de cordon est le plus important du monde (voir l'article d'émily Charrier et al.) $(\rightarrow)$. Les résultats cliniques sont excellents et

$(\rightarrow)$ p. 961

$(\rightarrow)$ p. 968

$(\rightarrow)$ p. 980

$(\rightarrow)$ p. 985

$(\rightarrow)$ p. 991

$(\rightarrow)$ p. 975 les chercheurs sont reconnus pour leurs travaux novateurs. Ce domaine de recherche présente un potentiel extraordinaire pour l'instauration de partenariats avec l'industrie, tel celui récemment concrétisé avec IBM, afin d'accélérer la recherche sur les causes et le traitement individualisé de la leucémie de l'enfant et d'autres maladies complexes en pédiatrie.

Comprendre, prévenir et résoudre les problèmes métaboliques importants allant de la période fœtale jusqu'au début de l'âge adulte est une préoccupation centrale de notre centre de recherche. Ce numéro vous présentera les travaux sur la prévalence et la distribution des maladies génétiques (voir l'article d'Anne-Marie Laberge et al.) ( $\rightarrow$ ), sur les effets fondateurs et la variabilité génétique au Québec (voir l'article de Claudia Moreau et al.) $(\rightarrow)$, ainsi que sur la dissection fonctionnelle du transport intestinal des lipides (voir l'article d'Emile Levy et al.) $(\rightarrow)$. Une équipe de chercheurs travaille aussi

$(\rightarrow)$ p. 1014 sur la cirrhose infantile, maladie spécifique des Amérindiens du Québec (voir l'article d'Andrea Richter et al.) $(\rightarrow)$.
La recherche sur l'avancement et le devenir en santé offre un éventail de compétences transversales aux chercheurs des autres thématiques, notamment en épidémiologie et en recherche évaluative. Ces domaines prennent toute leur importance au vu des préoccupations actuelles au sujet de la santé publique. Le souci d'équité et de justice sociale a toujours été présent dans les centres pédiatriques car la société a une responsabilité au regard de la vulnérabilité de l'enfant: pour nous, l'homme de demain est l'enfant d'aujourd'hui.

La compétence de nos chercheurs et la complémentarité des domaines prioritaires de recherche font déjà du CHU Sainte-Justine une centre d'excellence pour l'étude des problèmes de santé des mères et des enfants. L'ouverture sur le monde afin de favoriser des collaborations internationales complétant l'expertise de nos chercheurs et permettant de nous attaquer à des projets d'envergure est au centre des préoccupations de développement du Centre.

Le Centre de recherche du CHU Sainte-Justine se dote des ressources matérielles et humaines pour justifier ses ambitions, notamment en augmentant la superficie des espaces de recherche de plus de deux fois et en attirant un plus grand nombre de résidents et de stagiaires postdoctoraux avec des programmes de bourses conséquents. Notre but est de préparer la médecine de demain qui sera une médecine individualisée, et de hisser le Centre au rang de «référence mondiale» dans le domaine de la recherche «mèreenfant ». Pour ce faire, nous continuerons de favoriser l'intégration d'équipes multidisciplinaires s'attaquant aux grands problèmes de santé affectant les mères et les enfants en particulier dans les domaines de la périnatalité et du neurodéveloppement. Le dévouement de nos chercheurs est déjà une force de notre centre, le dynamisme et l'innovation de leurs travaux de recherche seront des atouts majeurs pour l'atteinte de notre objectif : I'amélioration de la santé de la mère et de l'enfant. $\diamond$

The medical research at Sainte-Justine: to grow healthy

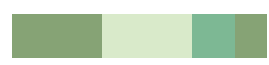

Guy A. Rouleau

Directeur du Centre de recherche, CHU Sainte-Justine, Chaire de recherche du Canada en génétique du système nerveux, Professeur titulaire, Faculté de médecine, Médecine et spécialités médicales, Université de Montréal. Centre de recherche, CHU Sainte-Justine, 3175, Côte Ste-Catherine, Montréal (Québec), H3T 1C6 Canada. guy.rouleau@umontreal.ca carine.daurat@crchum.qc.ca
TIRÉS À PART

G.A. Rouleau 